\title{
Experimental Study on the Three-Dimensional Unsteady Flow Characteristics of the Counter-Rotating Axial Flow Fan*
}

\author{
Lee-Sang $\mathrm{CHO}^{* *}$, Bong-Jun CHA*** and Jin-Soo CHO** \\ ** School of Mechanical Engineering, Hanyang University \\ 17 Haengdang-Dong, Seongdong-Gu, Seoul 133-791, Korea \\ E-mail: jscho@hanyang.ac.kr \\ *** Aero Engine Team, Korea Aerospace Research Institute \\ 45 Eoeun-Dong, Yuseong-Gu, Daejeon 305-806, Korea
}

\begin{abstract}
The counter-rotating axial flow fan shows that the complex flow characteristics with three-dimensional, viscous, and unsteady flow fields. For the understanding of the entire core flow in counter-rotating axial flow fan, it is necessary to investigate the three-dimensional unsteady flow field between the rotors. This information is also essential for the improvement of the aerodynamic characteristics, the reduction of the aerodynamic noise level and vibration characteristics of the counter-rotating axial flow fan. The purpose of this study is, therefore, to present the periodic characteristics of the blade passage flow, the wake and the tip vortex, which are utilized for the blade design data for the improvement of the aerodynamic characteristics, the reduction of the aerodynamic noise level and vibration characteristics of the counter-rotating axial flow fan. In this paper, the three -dimensional unsteady flow by the rotor-rotor interaction of the CRF were investigated at the design point(peak efficiency operating point). Unsteady flow fields in the CRF are measured at the cross-sectional planes of the upstream, between and downstream of each rotor using the $45^{\circ}$ inclined hot-wire probe. The stationary hot-wire technique used the $45^{\circ}$ inclined hot-wire probe, which rotates successively with 120 degrees increments about its own axis. And, the sampling data of unsteady flow fields were phase-locked averaged to remove the random components.
\end{abstract}

Key words: Counter-Rotating Axial Flow Fan, Three-Dimensional Unsteady Flow, Rotor-Rotor Interaction, $45^{\circ}$ Inclined Hot-Wire, Stationary Hot-Wire Technique, Phase-Locked Averaging Method

\section{Introduction}

The counter-rotating axial flow fan(CRF), which is a kind of two-stage axial-flow fan, has been a good solution for applications where high static pressure rise and volumetric flow rate are required ${ }^{(1)}$. The CRF consists of two counter-rotating rotors, which are a front rotor and a rear rotor, without stator blades ${ }^{(2)}$. Compared with commercial single-rotating axial flow fan, the CRF has higher performance characteristics and higher efficiency because the swirl velocity generated by the front rotor, which causes the energy loss, is recovered in the form of the static pressure by the rear rotor $^{(3)}$.

At the National Aeronautics and Space Administration (NASA) in USA, counterrotating systems ${ }^{(4)}$ were developed for the aircraft propulsion system because of its higher propulsion efficiency and lower specific fuel consumption, relatively. 
The aerodynamic characteristics of the CRF have investigated by several researchers. Kodama et al. ${ }^{(5)}$ showed that the fluid dynamic characteristics of the CRF are superior to those of the two-stage axial flow fan. But, the noise level of the CRF is higher compare with the two-stage axial flow fan. Shin et al. ${ }^{(6)}$ investigated the rotor-rotor interaction of counter-rotating unducted fan. The CRF shows the complex flow characteristics of three -dimensional, viscous, and unsteady flow fields. Therefore, the aerodynamic noise level of the CRF is increased due to rotor-rotor interaction by the two rotors with opposite rotational direction.

For the understanding of the entire core flow due to the rotor-rotor interaction of the $\mathrm{CRF}$, it is necessary to investigate the three-dimensional unsteady flow field between the rotors. This information is also essential to predict the aerodynamic and the acoustical characteristics of the CRF.

The purpose of this study is, therefore, to present the periodic characteristics of the blade passage flow, the wake and the tip vortex, which are utilized the blade design data for the improvement of the aerodynamic characteristics, the reduction of the aerodynamic noise level and vibration characteristics of the counter-rotating axial flow fan. In this present paper, the three-dimensional unsteady flow by the rotor-rotor interaction of the CRF were investigated at the design point(peak efficiency operating point). Unsteady flow fields in the CRF are measured at the cross-sectional planes of the upstream, between and downstream of each rotor using a single $45^{\circ}$ inclined hot-wire probe. The stationary hot-wire technique and the phase-locked averaged method for the $45^{\circ}$ inclined hot-wire probe were used to analyze the unsteady flow fields.

\section{Experimental Setup}

The experimental apparatus, as shown in Fig. 1, was set up for the investigation of three-dimensional unsteady flow characteristics of the CRF. Its total length is $7,650 \mathrm{~mm}$ and the diameter of the tested fan casing is $500 \mathrm{~mm}$. Test setups do not utilize an auxiliary fan and a duct system consists of the discharge duct only. A bell mouth, which is installed at the inlet of the tested fan, reduces the energy loss due to fluid friction and flow separation of the inlet flow. A conical damper is used to adjust the flow rate at the exit of the test duct.

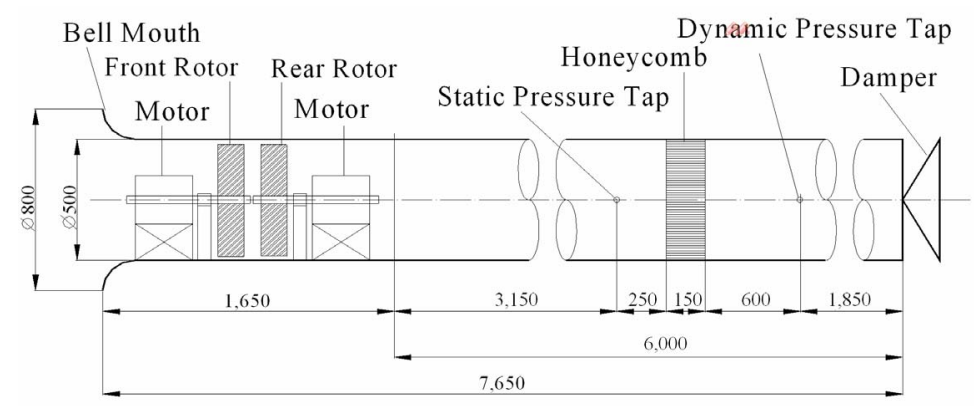

Fig. 1 Schematic diagram of the experimental setup, dimensions in $\mathrm{mm}$

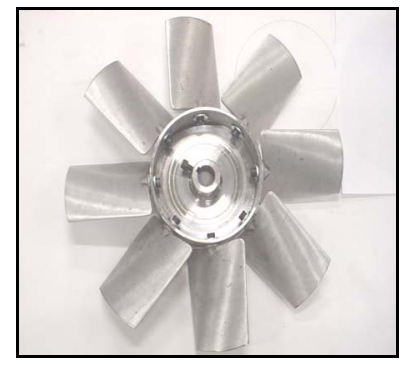

(a) Front rotor $\left(N_{B}=8\right)$

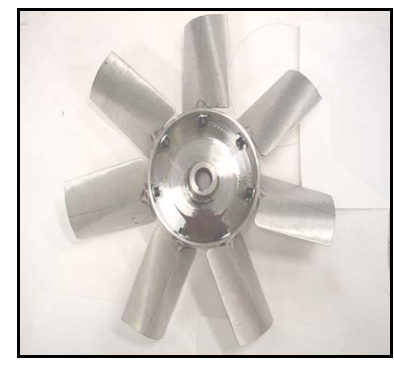

(b) Rear rotor $\left(N_{B}=7\right)$

Fig. 2 Front view of the front rotor and the rear rotor of the tested CRF 
The front rotor and the rear rotor of the CRF rotate in opposite direction and are driven by two separate electric motors. The front rotor of the CRF has eight blades and the rear rotor has seven blades as shown in Fig. 2.

Dimensions of the front rotor and the rear rotor are shown in Table 1. The diameter of the rotor is $497 \mathrm{~mm}$, and the hub-tip ratio is 0.4 . The stagger angle of the front rotor blade is 6 degrees lower than that of the rear rotor because of the increment of the relative velocity due to the opposite rotational direction of the rear rotor compared with the front rotor. The front rotor blades show opposite twist distributions compared with rear rotors.

Table 2 shows the peak efficiency operating condition obtained from the KS B $6311^{(7)}$. In this condition, the three-dimensional unsteady flow characteristics of the CRF were measured using the $45^{\circ}$ inclined hot-wire probe.

Table 1 Specifications for the front and rear rotors of the tested CRF

\begin{tabular}{|l|c|c|}
\hline & Front rotor & Rear rotor \\
\hline \hline Fan diameter & $500 \mathrm{~mm}$ & $500 \mathrm{~mm}$ \\
\hline Tip diameter & $497 \mathrm{~mm}$ & $497 \mathrm{~mm}$ \\
\hline Hub diameter & $199 \mathrm{~mm}$ & $199 \mathrm{~mm}$ \\
\hline Airfoil & \multicolumn{2}{|c|}{ NACA65-series } \\
\hline Camber angle & \multicolumn{2}{|c|}{$18 \mathrm{deg}}$. \\
\hline Stagger angle at mid span & $52.0 \mathrm{deg}$. & $58.0 \mathrm{deg}$. \\
\hline Solidity at mid span & 0.8 & 0.7 \\
\hline Number of blades & 8 & 7 \\
\hline
\end{tabular}

Table 2 Peak efficiency operating condition of the tested CRF

\begin{tabular}{|l|l|}
\hline Performance parameter & Operating condition \\
\hline \hline Total pressure rise $\left(\Delta P_{T}\right)$ & $37.5 \mathrm{mmH}_{2} \mathrm{O}(367.7 \mathrm{~Pa})$ \\
\hline Volumetric flow rate $(Q)$ & $2.68 \mathrm{~m}^{3} / \mathrm{s}(160.8 \mathrm{CMM})$ \\
\hline Fan efficiency $\left(\eta_{\text {fan }}\right)$ & $83.6 \%$ \\
\hline Rotational speed $(N)$ & $1750 \mathrm{rpm}$ \\
\hline
\end{tabular}

\section{Experimental Methods}

Three-dimensional unsteady flow fields of the CRF are investigated using a single $45^{\circ}$ inclined hot-wire probe. Many investigators have used the single-sensor hot-wire system for the measurement of three-dimensional flow fields and Reynolds stresses.

A single-sensor hot-wire measurement technique was developed by Whitfield, et al. ${ }^{(8)}$ and Hirsh and $\mathrm{Kool}^{(9)}$ has made attempts to extend these techniques to the measurement of turbulence intensities of the exit flow of a rotor.

\subsection{Geometry of a single $45^{\circ}$ inclined hot-wire probe}

Configuration of the single $45^{\circ}$ inclined hot-wire probe is shown in Fig. 4 . The inclined angle of the hot-wire sensor is represented by the unit vector $\vec{A}$ slanted at the probe angle $\theta_{0}$ to the $X$ axis. The probe coordinates $X, Y$ and $Z$ are fixed to the probe, the velocity vector is denoted by $\vec{V}$. The probe yaw angle $\theta_{y}$ changes by the turning angle while the pitch angle $\theta_{P}$ remains constant as the probe is rotated about its axis. The absolute flow angle $\alpha$ of the sensor is expressed in terms of the angles $\theta_{0}, \theta_{P}$ and $\theta_{y}$ as follows.

$\cos \alpha=\cos \theta_{0} \cdot \cos \theta_{p} \cdot \cos \theta_{y}+\sin \theta_{0} \cdot \sin \theta_{p}$

The relation between the velocity vector $\vec{V}$ and the hot-wire sensor is found in Ref. (9). 


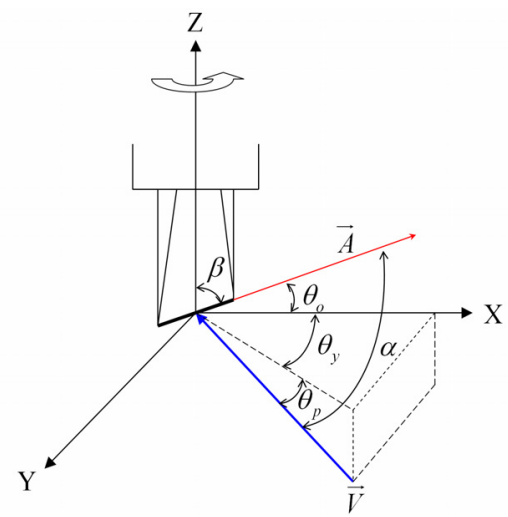

Fig. 4 Geometry of a $45^{\circ}$ inclined hot-wire probe

\subsection{Calibration of a $45^{\circ}$ inclined hot-wire probe}

The velocity calibration of the single $45^{\circ}$ inclined hot-wire probe was done with the sensor yaw angle $\theta_{y}$ equals to 90 degrees by the method of Grande and Kool ${ }^{(10)}$ in a wind tunnel $(0 \leq V \leq 60 \mathrm{~m} / \mathrm{s})$.

The turbulence level of the open type wind tunnel was 0.13 percent. The relation between the bridge voltage $(E)$ and the effective cooling velocity $\left(V_{e}\right)$ is expressed by the King's law ${ }^{(10)}$ as shown in Eq. (2).

$$
E^{2}=A+B \cdot V_{e}^{n}
$$

where the three coefficients $A, B$ and $n$ are determined from a velocity calibration data obtained using the hot-wire sensor normal to the flow.

The probe yaw angle calibration was carried out a large range of probe angles ( $-90 \leq \theta_{y} \leq+90$ degrees) at fixed values of velocity and angle between probe axis and velocity vector. The ratio of effective cooling velocity $V_{e}$ and actual velocity $V$ is expressed in terms of the angles $\theta_{0}, \theta_{P}$ and $\theta_{y}$ as follow ${ }^{(10)}$.

$$
\begin{aligned}
& \frac{V_{e}}{V}=\cos \psi \\
& \sin \psi=A_{2} \cos \theta_{p} \cos \left(\frac{\theta_{y}}{A_{1}}\right)+A_{2} \tan \theta_{0} \sin \theta_{p}
\end{aligned}
$$

where $\psi$ is a parameter in experimental probe angle calibration law.

The velocity and the probe yaw angle calibration results of the $45^{\circ}$ inclined hot-wire probe are fitted by the nonlinear curve fitting as shown in Fig. 5. The coefficients of the Eq. (2) and the Eq. (4) were determined by the least square fitting of the effective cooling velocity data.

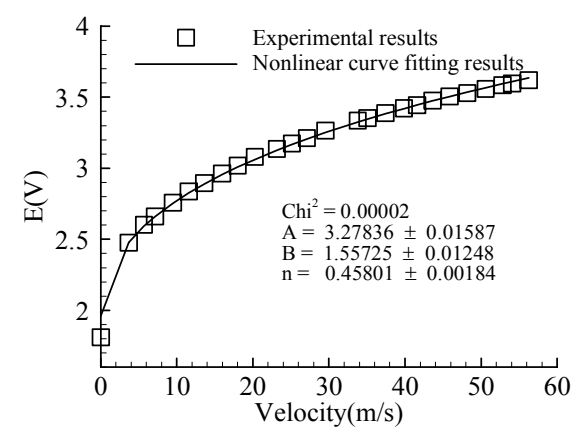

(a) Velocity calibration

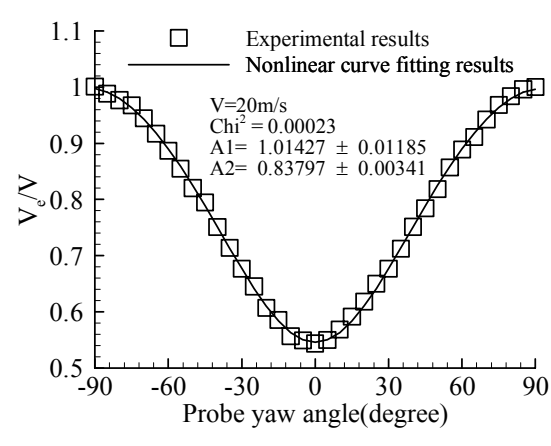

(b) Probe yaw angle calibration

Fig. 5 Calibration results of the $45^{\circ}$ inclined hot-wire probe 


\subsection{Measurement locations and technique}

Three-dimensional unsteady flow fields for the rotor-rotor interaction of the CRF were measured at cross-sectional planes of seven axial stations.

Figure 6 shows the schematic drawings of the CRF model and the single $45^{\circ}$ inclined hot-wire probe measurement locations. At the peak efficiency operating condition of the CRF, three-dimensional unsteady flow fields were measured at 23 points $\left(r / r_{t i p}=0.4\right.$ to 1.0) for the radial direction. The stationary hot-wire technique was used to the single $45^{\circ}$ inclined hot-wire probe, which rotates successively with 120 degrees increments about its own axis. The stationary hot-wire technique is presented by Grande and $\mathrm{Kool}^{(10)}$ in detail.

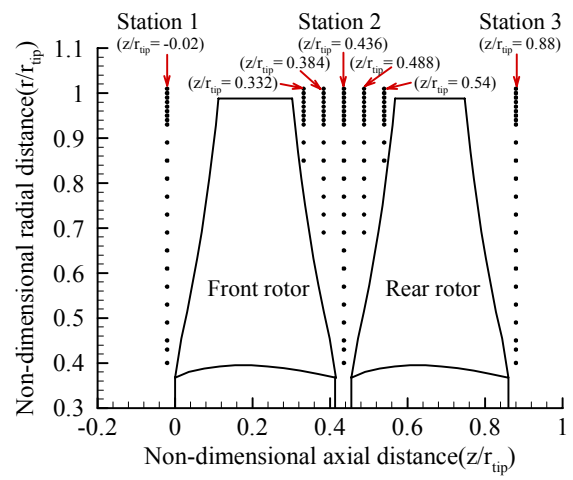

Fig. 6 Measurement locations for the three-dimensional unsteady flow fields of the CRF

\subsection{Data Acquisition and Data Reduction}

The data acquisition process of the single $45^{\circ}$ inclined hot-wire probe is shown in Fig. 7. The raw signal from the bridge output includes the random data as well as the phase information. In this technique, the hot-wire signal is triggered by a revolution frequency pulse in successive wave forms and are then averaged with one another so that only velocity components repeated every rotor revolution can be obtained.

The sampling data were taken for 4 blade passages of the front rotor $(3.5$ blade passages of the rear rotor), at each probe angle position and transferred to the computer. The sampling data were phase-locked averaged to remove the random component. At measuring points in Fig. 6, velocity data (100sets of 10,000 data) were taken and averaged.

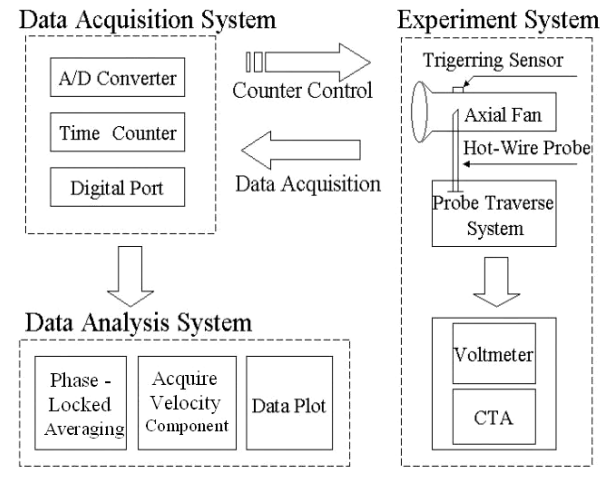

Fig. 7 Schematic diagram of the data acquisition and the data reduction for the CRF using the $45^{\circ}$ inclined hot-wire probe

\section{Results and Discussion}

The three-dimensional unsteady flow fields of the CRF are measured at seven axial stations(upstream of the front rotor, between the rotors and downstream of the rear rotor) using the single $45^{\circ}$ inclined hot-wire probe. 
Figure 8 shows non-dimensional phase-locked averaged velocity profiles at Station 1(upstream of the front rotor) of the CRF. Axial, radial and tangential velocity components are normalized by the mean axial velocity $V_{z 0}$ in the CRF. Also, rotor blade passage of the CRF is normalized by the parameter $Y(=2 r \theta / s)$. If the interval of the non-dimensional parameter $Y$ is 2 , it means 1 rotor blade passage. The measuring velocity profile data were 86 points for $4.3 \mathrm{~ms}$ per 1 blade passage of the front rotor. Rear rotor is 98 data points for $4.9 \mathrm{~ms}$ per 1 blade passage due to the number of rotor blades. Figure 8(a) shows the phase-locked averaged velocity profiles for 3 blade passages at the hub region of the front rotor inlet. The axial velocity is decreased compared with mean axial velocity $V_{z o}$ because of the flow separation by the installation of the front driving motor and the hub vortex by the rotation of the front rotor. The radial velocity is increased in the hub direction (negative direction) due to the flow contraction by the suction effect of the front rotor blade. The tangential velocity is increased at the front of the blade leading edge due to the unstable flow characteristics such as the flow separation and the hub vortex. Figure $8(\mathrm{~b})$ shows the phase-locked averaged velocity profiles at the mean radius of the front rotor inlet. At the mean radius, the axial, the radial and the tangential velocity profiles show more stable and periodical flow characteristics than those at the hub region. The axial velocity is more increased than the mean axial velocity $V_{z o}$. Flow patterns of the radial and the tangential velocity profiles are similar to those of the hub region. Figure 8(c) shows the phase-locked averaged velocity profiles at the tip region of the front rotor inlet. The axial velocity is decreased due to the boundary layer effect of the fan casing and the tip leakage flow. The radial and the tangential velocity profiles show the most unstable and unsteady flow characteristics compare with other position of the fan inlet.

Figure 9 shows phase-locked averaged velocity profiles for 3 blade passages of the front rotor at Station 2-3(between the rotors). As shown in Fig. 9(a), the axial velocity is decreased due to the flow separation and the boundary layer effect of the front rotor hub and the front motor. The radial velocity is not appeared the same as axial flow type turbomachinery. The tangential velocity is increased due to the swirl velocity by the rotation of the front rotor blade. At the whole, the phase-locked averaged velocity profiles of the front rotor outlet are periodically decreased by the wake flow characteristics due to the blockage effect at the trailing edge of the front rotor blade. Figure 9(b) shows the phase-locked averaged velocity profiles at the mean radius of the front rotor outlet. The axial velocity is more increased than that of the front rotor inlet due to flow contraction effect by the hub vortex and the casing boundary layer. The radial velocity profile is hardly appeared the same as that of the fan inlet flow. But, the tangential velocity is highly increased because of the increment of the absolute and the relative velocities by the rotation of the front rotor blade. Figure 9(c) shows the phase-locked averaged velocity profiles at the tip region of the front rotor outlet. The axial velocity is decreased in the form of the reverse

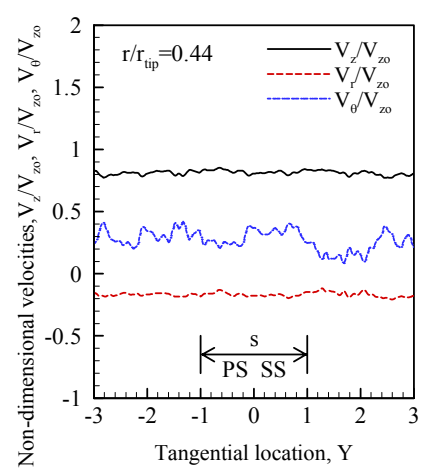

(a) hub region

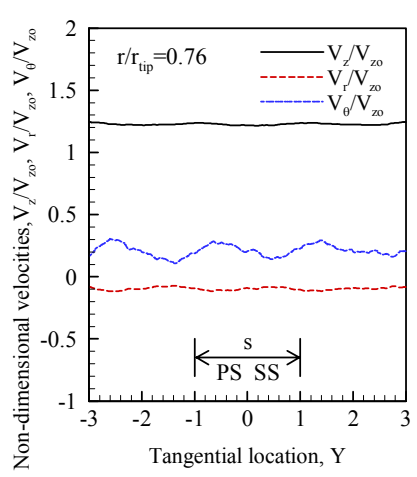

(b) mean radius region

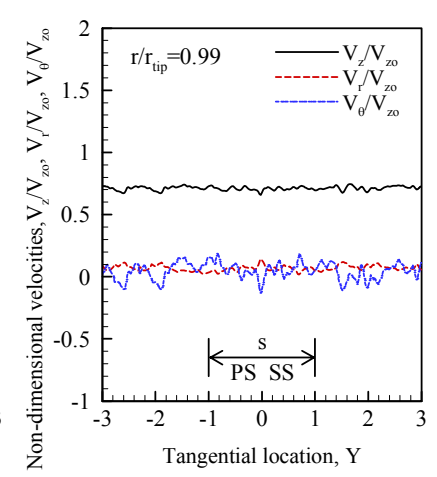

(c) tip region

Fig. 8 Phase-locked averaged velocity profiles at Station $1\left(z / r_{t i p}=-0.02, V_{z o}=16.6 \mathrm{~m} / \mathrm{s}\right)$ 
flow by the tip leakage flow and the tip vortex. At the trailing edge of the front rotor blade, the tangential velocity profile is decreased in the reverse direction by the tip vortex.

Figure 10 shows phase-locked averaged velocity profiles for 3 blade passages of the rear rotor at Station 3(downstream of the rear rotor). Figure 10(a) shows the phase-locked averaged velocity profiles at the hub region of the rear rotor outlet. The axial velocity is decreased in the reverse direction by the hub vortex and the flow separation of the rear rotor. Ideally, the tangential velocity have to remove is increased by the hub vortex due to the rotation of the rear rotor. But, the tangential velocity is increased by the hub vortex due to the rotation of the rear rotor. The tangential velocity component of the rear rotor induces the loss of the CRF. Figure 10(b) shows the phase-locked averaged velocity profiles at the mean radius of the rear rotor outlet. The axial velocity is more increased than that of the front rotor inlet and the outlet due to increment of the flow contraction effect. And, the removal of the tangential velocity component is not completed in spite of the rotation of the rear rotor. Figure 10 (c) shows the phase-locked averaged velocity profiles at the tip region of the rear rotor outlet. The axial velocity is decreased due to the effect of the leakage flow and the mixture of the front rotor and the rear rotor tip vortex. The tangential velocity is appeared in the rotational direction of the rear rotor because it is not tip vortex effect but the leakage flow effect of the rear rotor.

Figure 11 shows non-dimensional phase-locked averaged axial velocity profiles near the mean radius $\left(r / r_{t i p}=0.84\right)$ of the CRF. From the Station 2-1 to the Station 2-3, the phase -locked averaged velocity profiles are affected by the blade passage frequency of the front rotor. But, the Station 2-4 and the Station 2-5 are influenced by the blade passage frequency of the rear rotor. This result means the blockage effect of the front rotor and the suction effect of the rear rotor affected simultaneously between the rotors of the CRF.

Figure 12 shows the circumferential averaged axial and radial velocity vectors for the

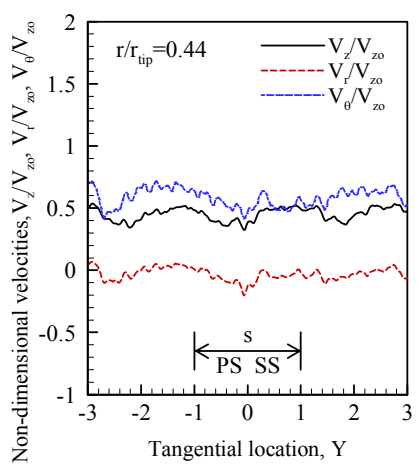

(a) hub region

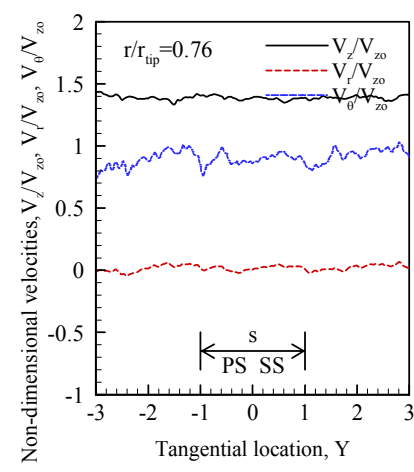

(b) mean radius region

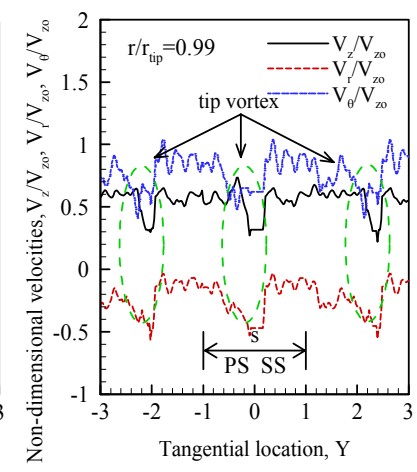

(c) tip region

Fig. 9 Phase-locked averaged velocity profiles at Station 2-3 $\left(z / r_{t i p}=0.436, V_{z o}=16.6 \mathrm{~m} / \mathrm{s}\right)$

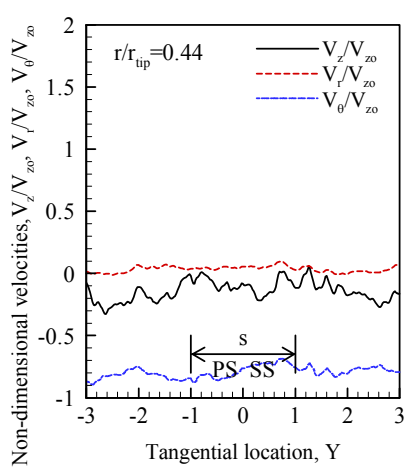

(a) hub region

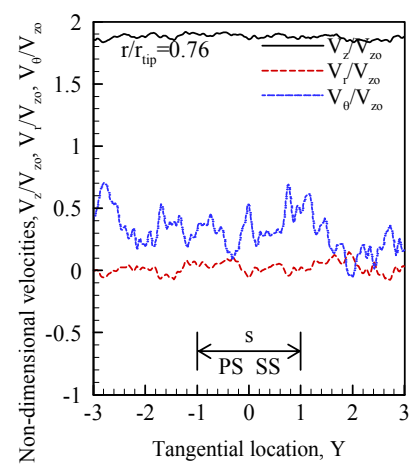

(b) mean radius region

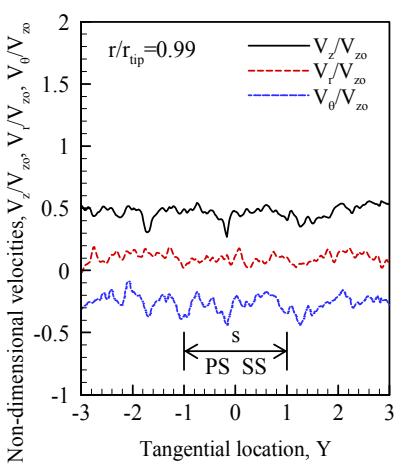

(c) tip region

Fig. 10 Phase-locked averaged velocity profiles at Station $3\left(z / r_{t i p}=0.88, V_{z o}=16.6 \mathrm{~m} / \mathrm{s}\right)$ 
through-flow fields in the CRF. As shown in Fig. 12, fan inlet flow field before the front rotor(Station 1) is relatively uniform except decrement of the axial velocity at the hub and the tip regions. At the tip region of the front rotor and the rear rotor, axial velocity is gradually decreased by the tip vortex not tip leakage flow. At the hub region of the front rotor and the rear rotor, axial velocity is gradually decreased and the radial velocity is increased due to the flow separation and the hub vortex.

Figure 13 shows the incidence angle and diffusion factor distributions at the front rotor and the rear rotor of the CRF. The incidence angle and the diffusion factor of the front rotor and the rear rotor are higher at the hub region due to the flow separation at the driving motor. This result means the pressure loss of the CRF is highly increased. Therefore, the front rotor and the rear rotor of the CRF are demanded the decline of the stagger angle at the hub region compared with the free vortex design condition for the increment of the fan efficiency.

Figure 14 shows the axial velocity contours, which are normalized by the rotational $\operatorname{speed}\left(U_{\text {tip }}=45.3 \mathrm{~m} / \mathrm{s}\right)$, at the each station of the CRF. Figure 14(a) shows the axial velocity contour for the cross flow pattern at the upstream of the front rotor. Axial velocity is decreased at the hub and the tip regions due to the boundary layer effect of the fan casing and due to the flow separation by the front motor as shown in Fig. 10(a). Figure 14(b) and (c) show the axial velocity contour at the downstream of the front rotor. At the blade tip region, axial velocity shows the reverse flow pattern in the axial direction by the tip vortex. This flow pattern is shown from the Station 2-1 $\left(z / r_{t i p}=0.332\right)$ to the Station 2-4 $\left(z / r_{t i p}=0.488\right)$. Figure $14(\mathrm{~d})$ shows the axial velocity contour between the rotors. At the mean radius, axial velocity is increased by the flow contraction effect due to the boundary layer of the fan casing and the hub vortex of the front motor compare with that of the Station 1(before the front rotor). As shown in Fig. 14(e), flow pattern of the axial velocity is

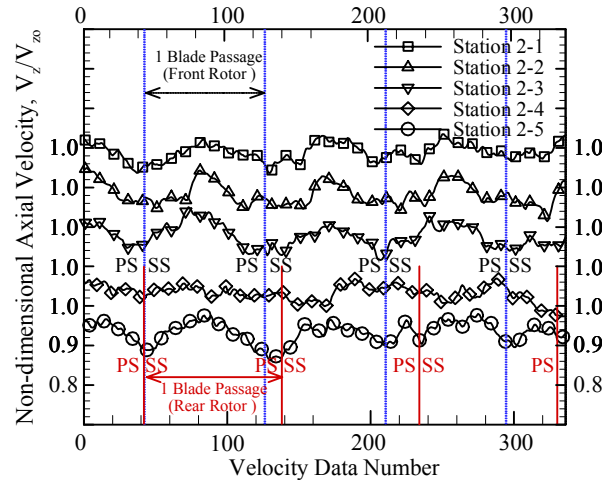

Fig. 11 Phase-locked averaged axial velocity profiles near the mean radius $\left(r / r_{t i p}=0.84\right)$

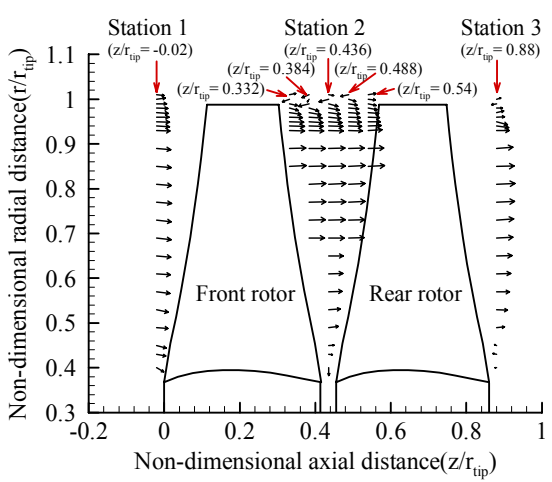

Fig. 12 Circumferential averaged axial and radial velocity vectors of the CRF

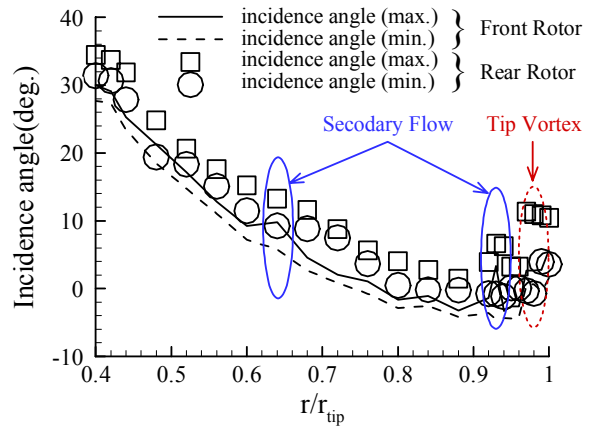

(a) Incidence angle distributions

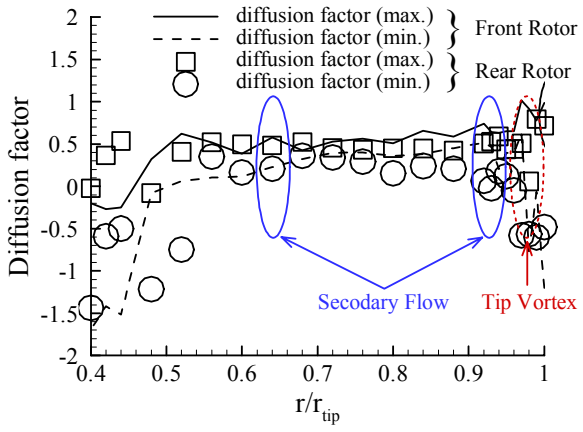

(b) Diffusion factor distributions

Fig. 13 Incidence angle and diffusion factor distributions of the CRF 
similar to the Station 2-2( $\left.z / r_{t i p}=0.384\right)$ but the tip vortex effect due to the rotation of the front rotor is decreased. Figure 14(f) shows the axial velocity contour before the rear rotor. Axial velocity is somewhat increased without reverse flow due to the suction effect of the rear rotor at the blade tip region. Figure $14(\mathrm{~g})$ shows the axial velocity contour at the downstream of the rear rotor. Axial velocity is higher at the mean radius due to the flow contraction effect. But, axial velocity is highly decreased at the hub region by the flow separation and the hub vortex of the front rotor and the rear rotor compare with that of the other axial station.

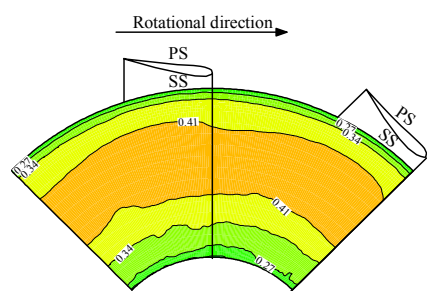

(a) Station 1( $\left.z / r_{\text {tip }}=-0.02\right)$

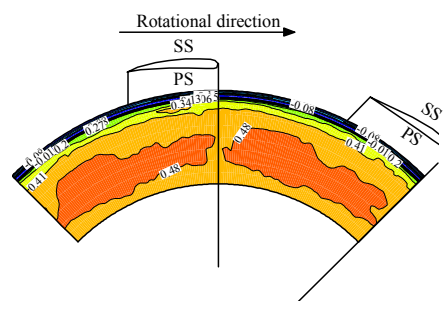

(c) Station 2-2( $\left.z / r_{\text {tip }}=0.384\right)$

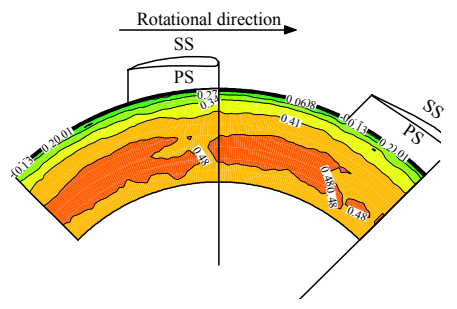

(e) Station $2-4\left(z / r_{t i p}=0.488\right)$

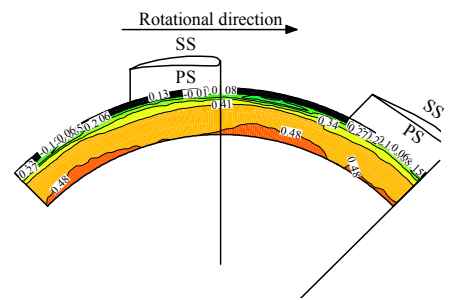

(b) Station 2-1( $\left.z / r_{t i p}=0.332\right)$

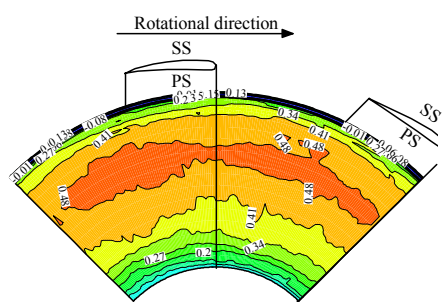

(d) Station 2-3( $\left.z / r_{t i p}=0.436\right)$

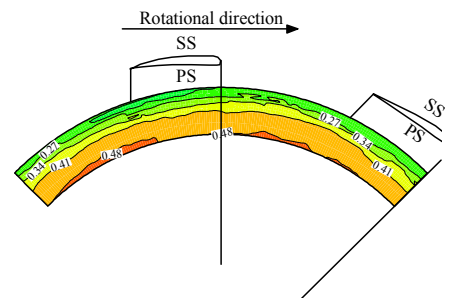

(f) Station 2-5( $\left.z / r_{\text {tip }}=0.54\right)$

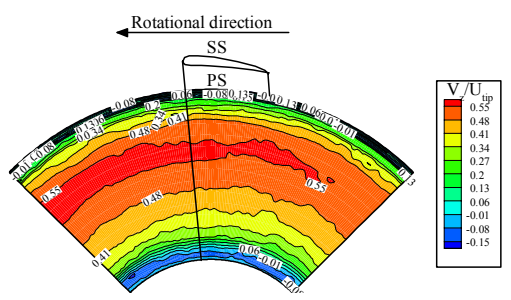

(g) Station 3( $\left.z / r_{\text {tip }}=0.88\right)$

Fig. 14 Non-dimensional axial velocity contours at each station of the CRF

\section{Conclusions}

From the experimental study on the three-dimensional unsteady flow characteristics of the CRF, the results are summarized as follows.

(1) The phase-locked averaged velocity profiles of the CRF are analyzed by means of the stationary unsteady measurement technique. At the whole, the axial velocity is 
decreased due to the flow separation by the installation of the front driving motor and the hub vortex by the rotation of rotors at the hub region of the front rotor and the rear rotor. At the mean radius of the front rotor inlet and the outlet, the axial, the radial and the tangential velocity profiles show more periodical flow characteristics than those of the hub region. At the tip region of the front rotor inlet and the outlet, the axial velocity is decreased by the boundary layer effect of the fan casing and the tip leakage flow. The radial and the tangential velocity profiles show the most unstable and unsteady flow characteristics compared with other position of rotors.

(2) From the Station 2-1 to the Station 2-3 of the between the rotors of the CRF, the phase-locked averaged velocity profiles are affected by the blade passage frequency of the front rotor. But, the Station 2-4 and the Station 2-5 are influenced by the blade passage frequency of the rear rotor. This result means the blockage effect of the front rotor and the suction effect of the rear rotor affected simultaneously between the rotors of the CRF.

(3) For the axial velocity contour for the cross-flow pattern of the CRF, the axial velocity is highly decreased due to the boundary layer effect by the fan casing and the blade tip vortex at the blade tip region of the front and the rear rotors. At the hub region, axial velocity is decreased due to the flow separation at the driving motor. This result induces the increment of the incidence angle and the diffusion factor of the front rotor and the rear rotor. Therefore, the front rotor and the rear rotor of the CRF are demanded the decline of the stagger angle at the hub region compared with the free vortex design condition for the increment of the fan efficiency.

\section{Acknowledgement}

This study has been supported by the KARI under KHP Dual-Use Development Program funded by the Ministry of Knowledge Economy.

\section{References}

(1) Bleier, F. P., Fan Handbook, (1998), pp. 4.28-4.38, McGraw-Hill, New York.

(2) Wallis, R. A., Axial Flow Fans and Ducts, (1983), pp. 309, John Wiley \& Sons Inc.

(3) Cho, J. S. and Cho, L. S., Experimental Study on the Aerodynamic Characteristics of a Two-Stage and a Counter-Rotating Axial Flow Fan, Transactions of the Korean Society of Mechanical Engineers, Vol. 23, No. 8(B), (2001), pp. 1048-1062.

(4) Strack, W. C., Knip, G., Weisbrich, A. L., Godston, J., and Bradly, E., Technology and Benefits of Aircraft Counter Rotation Propellers, NASA TM 82983, (1982).

(5) Kodama, Y., Hayashi, H., Fukano, T. and Tanaka, K., Experimental Study on the Characteristics of Fluid Dynamics and Noise of a Counter-Rotating Fan, Transactions of the Japan Society of Mechanical Engineers, Vol. 60, No. 576(B), (1994), pp. 2764-2779.

(6) Shin, H. W., Whitfield C. E. and Wisler D. C., Rotor-Rotor Interaction for CounterRotating Fans, Part 1: Three-Dimensional Flow field Measurements, AIAA Journal, Vol. 32, No. 11, (1994), pp. 2224-2233.

(7) KS B 6311, Testing Methods for Turbo Fans and Blowers, (1982), Korean Standards Association.

(8) Whitfield, C. E., Kelly, J. C. and Barry, B., A Three Dimensional Analysis of Rotor Wakes, Aero Quarterly, Vol. 23(Part 4), (1972), pp. 285-300.

(9) Hirsh, C. and Kool, P., Measurement of the Three-Dimensional Flow Field Behind an Axial Compressor Stage, Journal of Engineering for Power, Vol. 99, (1977), pp. 168-180.

(10) Grande, G, and Kool, P., An Improved Experimental Method to Determine the Complete Reynolds Stress Tensor with a Single Rotating Slanting Hot Wire, The Institute of Physics, Vol. 14, (1981), pp. 196-201. 